\section{BMJ Open} Ophthalmology

\title{
Assessment of objective ocular cyclodeviation under monocular condition and binocular condition using fundus photography
}

Noriaki Murata (D) , Haruo Toda, ${ }^{1}$ Haruna Amaki, ${ }^{1}$ Kanako Suzuki, ${ }^{1}$ Yumi Nagai, ${ }^{1}$ Yuna Omiya, ${ }^{1}$ Tomomi Kurashima, ${ }^{1}$ Sachiko Udagawa, ${ }^{2}$ Shinji Ohkubo ${ }^{2}$

To cite: Murata $\mathrm{N}$, Toda $\mathrm{H}$, Amaki H, et al. Assessment of objective ocular cyclodeviation under monocular condition and binocular condition using fundus photography. BMJ Open Ophthalmology 2020;5:e000595. doi:10.1136/ bmjophth-2020-000595

Received 8 August 2020 Revised 13 November 2020 Accepted 14 December 2020

\section{Check for updates}

(c) Author(s) (or their employer(s)) 2020. Re-use permitted under CC BY-NC. No commercial re-use. See rights and permissions. Published by BMJ.

${ }^{1}$ Department of Orthoptics and Visual Sciences, Niigata University of Health and Welfare, Niigata, Niigata, Japan

${ }^{2}$ Department of Ophthalmology and Visual Science, Kanazawa University Graduate School of Medical Sciences, Kanazawa, Ishikawa, Japan

Correspondence to Noriaki Murata; murata@nuhw. ac.jp

\section{ABSTRACT}

Objective The relationship between retinal structure and function of glaucomatous eyes has attracted a great deal of research attention. However, visual field tests are conducted under monocular condition, and ophthalmic imaging was performed in patients without occlusion. We aimed to assess the objective ocular cyclodeviation between monocular occlusion and binocular conditions using fundus photography.

Methods and analysis This study included 76 healthy participants. We obtained six photos of the right eye of each patient using fundus photography. Three of the photographs were taken under monocular conditions, and the other three, under binocular conditions. We measured the optic disc margin-fovea angle (MFA) of the line connecting one point of the disc limbus and the fovea. One-way repeated analysis of variance was used to compare the angles under both conditions. We also examined the direction of ocular rotation under the binocular condition regarding the monocular condition.

Results The MFAs were $12.12^{\circ} \pm 3.83^{\circ}$ and $12.19^{\circ} \pm 3.95^{\circ}$ under the monocular and binocular conditions, respectively. There was no significant difference in both MFAs $(F=1.19, \mathrm{p}=0.28)$. The mean cyclodeviation was $0.07^{\circ} \pm 0.80^{\circ}$ (range: $-2.40^{\circ}$ to $\left.+2.75^{\circ}\right)$. A total of 38 eyes showed excycloduction, while another 38 showed incycloduction.

Conclusion Significant cyclodeviation did not occur regardless of the existence of an occlusion. When examining the relationship between retinal structure and function, the difference in rotation angle under both conditions need not be taken into consideration if the other disease did not cause pathological cyclodeviation.

\section{INTRODUCTION}

The relationship between retinal structure and function should be evaluated during clinical follow-up and in the study of glaucoma. ${ }^{1}$ The structure of a glaucomatous eye is mainly assessed using fundus photography and optical coherence tomography (OCT) and measured in the binocular opening situation. The visual field (VF) sensitivity is generally determined by standard automated perimetry $(\mathrm{SAP})^{2}$; it usually requires the

\section{Significance of the study}

What is already known about this subject?

With regard to the relationship between structure and function in patients with glaucoma, attention has been focused on the possibility of improving the diagnosis by correcting the anatomical position of the fovea and optic disc centre. Several studies reported that the correction based on the positional relation of the disc and fovea did not significantly affect the diagnostic performance.

\section{What are the new findings?}

D The measurement conditions differ from the viewpoint of the presence or absence of one eye occlusion in the two tests: ocular structural imaging and visual field testing. We evaluated whether or not a difference exists in the ocular cyclodeviation due to the difference in the measurement condition using an objective cyclic angle and found that there was no significant change.

How might these results change the focus of research or clinical practice?

The cyclodeviation due to the difference in monocular or binocular viewing conditions need not be considered when performing both visual field testing and ophthalmic imaging.

untested eye to be occluded. Assuming that ocular rotation has occurred due to the presence of cyclophoria since the measurement conditions differ between the structure examination and VF, the overlay of both data will be misaligned. When observing the relationship between the test pointwise of the $\mathrm{VF}$ and the local retinal thickness, ${ }^{3-5}$ the effect of rotational misalignment may become greater as the distance from the fovea to the periphery increases. To our best knowledge, no study has evaluated the objective ocular cyclodeviation during monocular occlusion. Therefore, the possibility of cyclodeviation due to occlusion cannot be eliminated. 
Several methods have been used for measuring the objective cyclodeviation including artificial markers such as physical markers, corneal tattoo, scleral markings, ${ }^{67}$ search coil, ${ }^{8}$ tracking system on an excimer laser, ${ }^{9}$ the iris pattern imaging ${ }^{10}$ and fundus imaging. ${ }^{11-19}$ In this study, we used fundus photography to reproduce the conditions similar to that during OCT. Thereby, we aimed to assess the differences and variations in the angle of cyclodeviation under binocular opening situation and monocular occlusion.

\section{MATERIALS AND METHODS \\ Participants}

We examined 76 right eyes of 76 healthy participants (19 men and 57 women) who had no eye disease except for refractive error. Written informed consent was obtained from all participants after receiving an explanation of the procedures in this study. The mean \pm SD for age was $21.03 \pm 1.06$ years, with a range of $18-24$ years. We performed an ophthalmic examination, which included assessment of best-corrected visual acuity (BCVA) using a $5 \mathrm{~m}$ Landolt chart, refraction, keratometry, VF testing using the 24-2 Swedish Interactive Threshold AlgorithmFast Strategy (Humphrey Field Analyzer; Carl Zeiss Meditec, Dublin, California, USA) for all participants. All recruited participants had BCVA better than \pm 0.00 logarithm of the minimum angle of resolution, which is equivalent to a Snellen's acuity of $20 / 20$, and no VF defect in both eyes. The definition of VF defects was based on the Anderson and Patella criteria. ${ }^{20}$ We performed a cover test and measured the subjective angle using a major amblyoscope to assess for apparent strabismus and subjective cyclodeviation.

\section{Fundus photography}

The images were taken using a non-mydriatic fundus camera nonmyd WX (KOWA, Tokyo, Japan) with $45^{\circ}$ of view. The participants' faces were placed on the chin rest of the equipment under monocular condition, and a paste-type eyepatch was applied. The participants were instructed to fixate their eyes on the internal fixation lamp, and three fundus photographs were taken. The eye patches were removed with great care so that the midline of the face did not change, and then three photos were taken under binocular condition. Since the experiment was performed under the non-mydriatic condition in a dark room, it took $30 \mathrm{~s}$ to $1 \mathrm{~min}$ from one flash to the natural mydriasis and about $9 \mathrm{~min}$ in the total examination. The participants' head position remained unchanged throughout the assessment. For this reason, all participants underwent fundus photography in the order of the monocular condition and then the binocular condition. We did not fix the position of the participants' head with a belt to reproduce the circumstances of daily medical practice (figure 1 ).

\section{Data processing}

We used Image $\mathrm{J}^{21}$ to analyse the fundus images. The photographs were imported into Image $\mathrm{J}$ and analysed

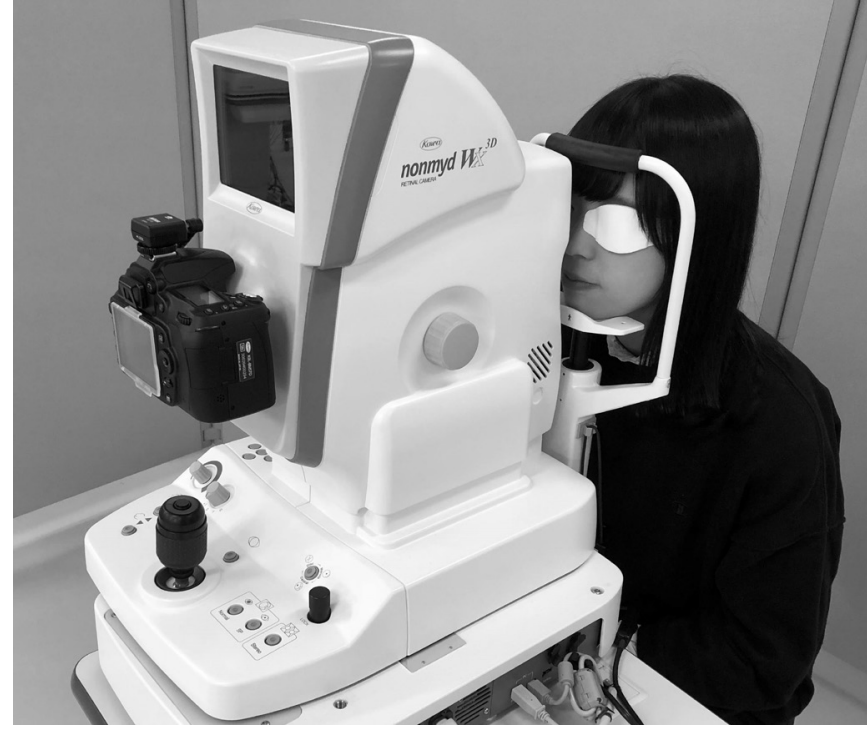

Figure 1 Fundus photography setup during the experiment. We did not use a fixed head belt to replicate the routine medical practice. The obstruction was always performed with a pasting type eyepatch, and great care was taken when the head position was adjusted while removing the occluder. We waited for the natural mydriasis to occur after flash. even during that time, the participants were asked to maintain a fixed head position.

using the angle tool (figure 2). In this study, we did not measure the absolute angle because we focused only on the changes in the angle of cyclodeviation under the two conditions. Therefore, we did not measure the disc-foveal angle ${ }^{14-19}$ which required identification of the centre of the optic disc. Instead, the narrowest and clearest vessels on the optic disc margin were selected for each participant, and a straight line was drawn at the intersection (triangle) and fovea (asterisk) between the vessel and papillary limbus. The angle between this line (solid line) and the horizontal line (dotted line) crossing the fovea was calculated. In this study, this measurement value was referred to as the 'optic disc margin-foveal angle' (MFA). The MFAs had a positive value because we chose the blood vessels running in the upper region rather than those in the optic disc centre. At first, on every fundus photograph, three measurements were repeated and averaged. This procedure performed three authors independently. Then, the gross average across the data from three authors was calculated to minimise the measurement bias. Finally, the MFA of participants under each condition was determined as the mean of the angle of three fundus photographs.

\section{Statistical analysis}

Statistical analysis was performed using $\mathrm{R}$ version V.3.5.1 (http://www.r-project.org/) and anovakun V.4.8.2 (http://riseki.php.xdomain.jp/index.php?ANOVA\% $\mathrm{E} 5 \% 90 \% 9 \mathrm{~B})$. We used the one-way repeated analysis of variance (ANOVA) to evaluate the difference between MFA under the monocular condition and MFA under the binocular condition. Pearson's correlation coefficient 


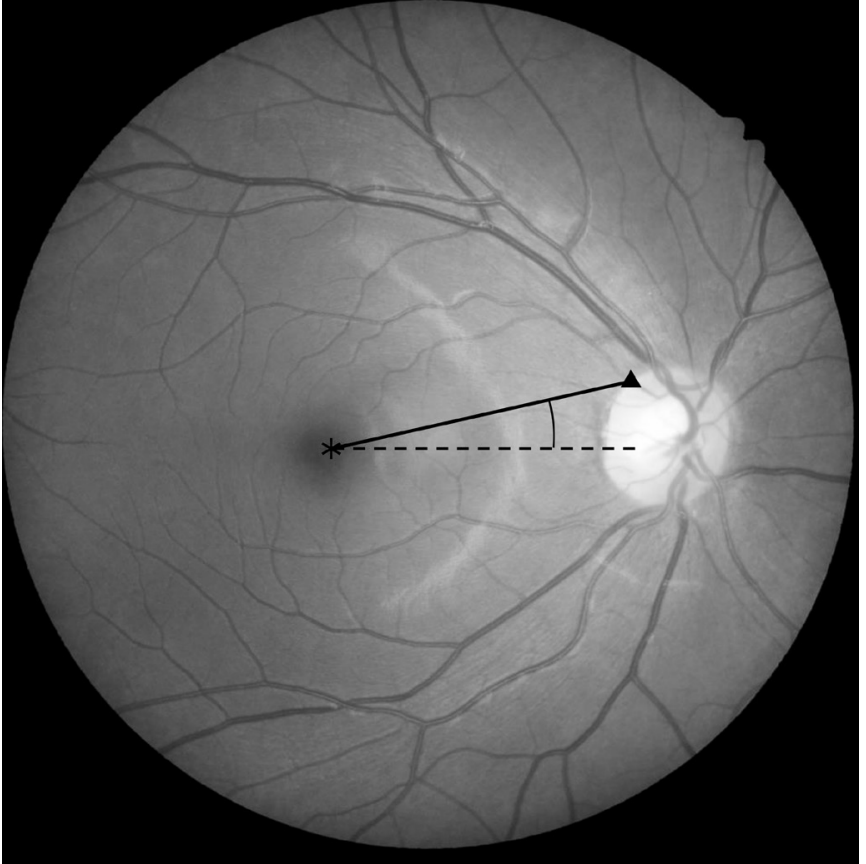

Figure 2 Measurement of optic disc margin-fovea angle. First, we selected the most apparent and narrowest vessels on the optic disc and identified the intersection with the limbus of the disc (triangle). Then, both the triangle and the fovea (asterisk) were connected with a straight line (solid line), and the angle between the solid line and the horizontal line across the fovea was measured (dotted line). The intersection was set above the centre of the optic disc.

was calculated to assess the correlation between the measured values under both conditions. $P$ values of $<0.05$ were considered significant. We calculated the amount of cyclodeviation under both binocular and monocular conditions. A positive value indicates excyclodeviation, and a negative value indicates incyclodeviation. Statistical values were presented as mean $\pm \mathrm{SD}$ unless otherwise specified.

\section{Patient and public involvement}

This research was conducted in connection with a part of the educational courses at Niigata University of Health and Welfare. The students participated in the preparations and measurements of the study were included in the coauthors. The other students were involved in the general discussion with us. No patients and the general public were involved in this study.

\section{RESULTS}

The mean refractive error (spherical equivalent) was $-1.79 \pm 2.02 \mathrm{D}$ (range: -7.25 to $+2.00 \mathrm{D}$ ). The MFAs were $12.12^{\circ} \pm 3.83^{\circ}$ under the monocular condition and $12.1^{\circ} \pm 3.95^{\circ}$ under the binocular condition. Figure 3 shows a histogram of the distribution of the MFA difference under both conditions. There was no significant difference between the two states as a result of one-way repeated ANOVA $(F=1.19, \mathrm{p}=0.28)$. The mean amount of cyclodeviation under the binocular condition as a

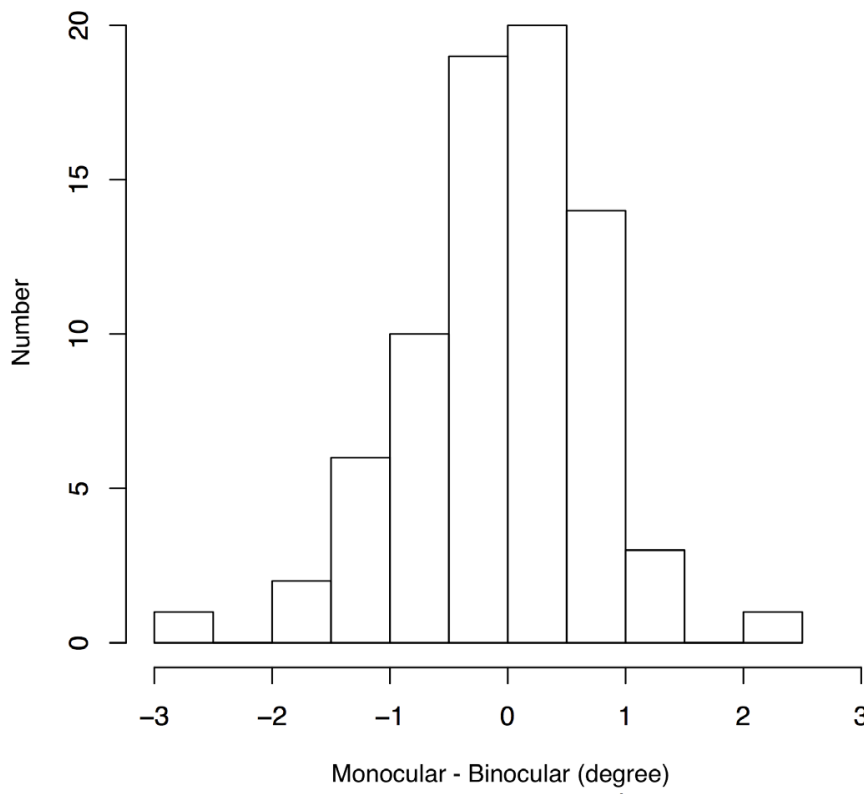

Figure 3 Distribution of optic disc margin-foveal angle differences in two conditions. The amount of cyclodeviation under both the binocular and monocular conditions are shown. Positive values indicate excycloduction, while negative values indicate incycloduction. The same number of participants showed incyclic shifts and exocyclic shifts. Removing the occlusion had no consistent effect on the direction of rotation.

reference on the monocular condition was $0.07^{\circ} \pm 0.80^{\circ}$ (range: $-2.40^{\circ} \pm 2.75^{\circ}$ ). A total of 38 eyes showed excyclodeviation, while another 38 showed incyclodeviation. Figure 4 shows a scatter plot of the MFA under both conditions. A significantly high correlation was observed between the measured values under both conditions $(r=0.98, \mathrm{p}<0.01)$. The MFA measurement under both conditions suggested that there was no difference in the cyclodeviation.

\section{DISCUSSION}

In this study, we assessed whether or not the ocular cyclodeviation would occur due to the occlusion during VF testing, which is routinely performed as part of the medical care. Results showed that there was no difference between the two conditions. Glaucoma is a neurodegenerative disease characterised by a slow and progressive degeneration of retinal ganglion cells, causing visual impairment, thinning of the retinal nerve fibre layer (RNFL), and enlargement of the optic disc cup. All of these are associated with the loss of retinal ganglion cell axons. ${ }^{22-24}$ Recently, with regard to the evaluation of retinal structure, OCT was performed for the threedimensional measurement of retinal thickness. The analysis of the circumpapillary RNFL, ${ }^{25}$ ganglion cell complex $^{27}$ and ganglion cell-inner plexiform layer ${ }^{28}$ in the macula enables the comprehensive observation of both function and structure in daily medical practice to determine the progress of glaucoma. Chauhan et $a t^{29}$ mentioned the positional relationship between the disc 


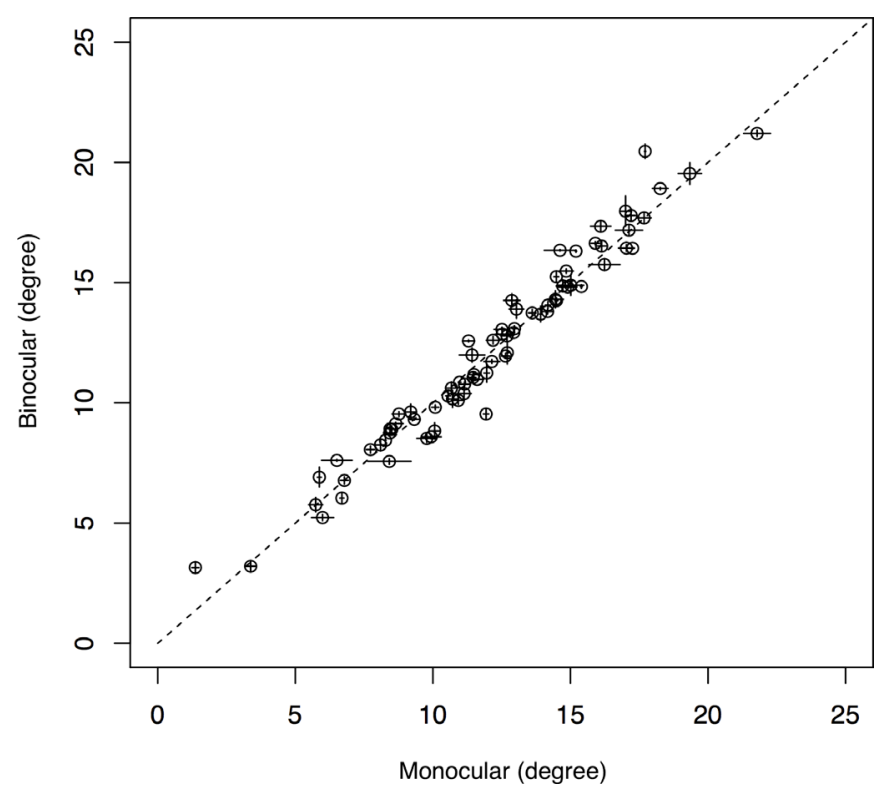

Figure 4 Scatter plots of optic disc margin-foveal angle. We only analysed the amount of change under both conditions because the blood vessel running was different for each participant. The magnitude of the angle of each participant was not significant. Error bars indicate the SE.

centre and fovea and stated that the correction of the anatomical horizontal line and the horizontal line of the fundus photograph may increase the ability to diagnose OCT parameters concerning the positional relationship between disc and fovea. Several studies have used the OCT parameter correction technique to determine the positional relationship between disc and fovea. ${ }^{17} 183031$ However, to our knowledge, no study has reported the usefulness of improving the ability to diagnose glaucoma by correcting the disc-fovea angle. Mwanza et $a l^{17}$ performed an RNFL profile correction based on the disc-fovea angle for healthy individuals, myopic individuals and patients with glaucoma. However, the thickness, specificity and glaucoma diagnostic performance of RNFL parameters remained inconsistent. Mayama et $a l^{30}$ provide a comparison between with and without correction of inclination of performance of the optimum or suboptimum condition in detecting early-stage glaucoma in the study of grid-wise macular inner retinal layer thickness. As a result, they reported that the diagnostic capability was not improved under any condition by compensation of the inclination of the disc-fovea line. Moreover, it does not match the estimation of the VF defect if cyclodeviation occurred in the eye with eyepatch during the actual VF testing, even if the rotation angle correction on the OCT images is sufficient. From a different viewpoint than the previous studies, the present study suggests that unilateral occlusion does not induce cyclodeviation in normal participants who have no abnormalities in binocular vision. To summarise, correcting natural cyclodeviation caused by occlusion need not be taken into consideration when evaluating the structure and function of the glaucomatous eyes. Functional imaging could be used to follow up on the progression of glaucoma or predict VF impairment unless a patient developed complications that can cause pathological cyclodeviation, such as superior oblique palsy.

With regard to the treatment of strabismus, the occlusion of one eye is referred to as the fusion-free position. ${ }^{32}$ Although cyclophoria becomes apparent in the fusionfree position, the objective cyclodeviation was not observed in the healthy participants included in this study. This experiment was performed in a dark room, and the participant was instructed to fixate with a fixation lamp. Accordingly, the fusion-free position was reproduced under both conditions, which may be the reason why the ocular rotation angle remained unchanged. It may be unnecessary to ponder this point because the fusion-free position seems to be reproduced for patients in both VF testing and imaging examination in actual clinical practice.

Our study has several limitations. First, the study was only performed in participants aged around 20 years. Miyata $e t a l^{16}$ evaluated the age-related changes in DFA in the Nagahama study and reported that older people had larger DFA than younger people and tended to exhibit exocyclic rotation according to age. Further studies are warranted among participants with various ages and glaucoma patients to examine the incidence of cyclodeviation. Second, while we found no significant cyclodeviation within $9 \mathrm{~min}$, sometimes the measurement of SAP requires over $10 \mathrm{~min}$ depending on the degree of VF defect and the strategy employed. In such a case, cyclodeviation may change due to fatigue. A new approach using a fundus automated perimeter may help evaluate the rotation angle variability during the clinical VF testing.

In conclusion, we quantitatively analysed the objective ocular cyclodeviation under the monocular condition and the binocular condition using Image J. There was no statistically significant difference in the amount of deviation with or without occlusion. Although the measurement conditions differ based on the presence or absence of occlusion between ophthalmic imaging and VF testing, the degree of cyclodeviation need not be considered when performing both inspections.

Correction notice This article has been corrected since it first published. The provenance and peer review statement has been included.

Acknowledgements The authors would like to thank Enago (www.enago.jp) for the English language review.

Contributors NM is the first author who completed the analysis and prepared this paper. HT, SU and SO reviewed the analysis and contributed to the content of this paper. HA, KS, YN, YO and TK collected the data, reviewed and contributed to the content of this paper.

Funding This study was supported by a Grant-in-Aid for Scientific Research from the Japan Society for the Promotion of Science (Kakenhi, 18K17770) (https://www. jsps.go.jp/j-grantsinaid/).

Competing interests None declared.

Patient and public involvement Patients and/or the public were not involved in the design, or conduct, or reporting, or dissemination plans of this research.

Patient consent for publication Obtained. 
Ethics approval This prospective study was approved by the Ethics Committee of Niigata University of Health and Welfare. All the procedures used conformed to the tenets of the Declaration of Helsinki.

Provenance and peer review Not commissioned; externally peer reviewed.

Data availability statement Data are available upon request.

Open access This is an open access article distributed in accordance with the Creative Commons Attribution Non Commercial (CC BY-NC 4.0) license, which permits others to distribute, remix, adapt, build upon this work non-commercially, and license their derivative works on different terms, provided the original work is properly cited, appropriate credit is given, any changes made indicated, and the use is non-commercial. See: http://creativecommons.org/licenses/by-nc/4.0/.

ORCID iD

Noriaki Murata http://orcid.org/0000-0001-8241-7851

\section{REFERENCES}

1 Harwerth RS, Wheat JL, Fredette MJ, et al. Linking structure and function in glaucoma. Prog Retin Eye Res 2010;29:249-71.

2 Johnson CA. Standardizing the measurement of visual fields for clinical research: guidelines from the eye care technology forum. Ophthalmology 1996;103:186-9.

3 Ajtony C, Balla Z, Somoskeoy S, et al. Relationship between visual field sensitivity and retinal nerve fiber layer thickness as measured by optical coherence tomography. Invest Ophthalmol Vis Sci 2007;48:258-63.

4 Ohkubo S, Higashide T, Udagawa S, et al. Focal relationship between structure and function within the central 10 degrees in glaucoma. Invest Ophthalmol Vis Sci 2014;55:5269-77.

5 Takahashi M, Omodaka K, Maruyama K, et al. Simulated visual fields produced from macular RNFLT data in patients with glaucoma. Curr Eye Res 2013;38:1133-41.

6 Clarke AH, Engelhorn A, Hamann C, et al. Measuring the otolithocular response by means of unilateral radial acceleration. Ann NY Acad Sci 1999;871:387-91.

7 Migliaccio AA, Macdougall HG, Minor LB, et al. Inexpensive system for real-time 3-dimensional video-oculography using a fluorescent marker array. $J$ Neurosci Methods 2005;143:141-50.

8 Collewijn H, van der Mark F, Jansen TC. Precise recording of human eye movements. Vision Res 1975;15:447-IN5.

9 Swami AU, Steinert RF, Osborne WE, et al. Rotational malposition during laser in situ keratomileusis. Am J Ophthalmol 2002;133:561-2.

10 Ong JKY, Haslwanter T. Measuring torsional eye movements by tracking stable iris features. J Neurosci Methods 2010;192:261-7.

11 Ehrt O, Boergen KP. Scanning laser ophthalmoscope fundus cyclometry in near-natural viewing conditions. Graefes Arch Clin Exp Ophthalmol 2001;239:678-82.

12 Kushner BJ, Hariharan L. Observations about objective and subjective ocular torsion. Ophthalmology 2009;116:2001-10.

13 Madigan WP, Katz NN. Ocular torsion-direct measurement with indirect ophthalmoscope and protractor. J Pediatr Ophthalmol Strabismus 1992;29:171-4.
14 Choi JA, Kim J-S, Park H-YL, et al. The foveal position relative to the optic disc and the retinal nerve fiber layer thickness profile in myopia. Invest Ophthalmol Vis Sci 2014;55:1419-26.

15 Jonas RA, Wang YX, Yang H, et al. Optic Disc - Fovea Angle: The Beijing Eye Study 2011. PLoS One 2015;10:e0141771.

16 Miyata M, Yoshikawa M, Ohtsuki H, et al. Age-Related change and sex difference over 60S in disc-fovea angle in Japanese population: the Nagahama study. Acta Ophthalmol 2018;96:e840-5.

17 Mwanza J-C, Lee G, Budenz DL. Effect of adjusting retinal nerve fiber layer profile to Fovea-Disc angle axis on the thickness and glaucoma diagnostic performance. Am J Ophthalmol 2016;161:12-21.

18 Resch H, Pereira I, Hienert J, et al. Influence of disc-fovea angle and retinal blood vessels on interindividual variability of Circumpapillary retinal nerve fibre layer. Br J Ophthalmol 2016;100:531-6.

19 Yamashita T, Sakamoto T, Terasaki H, et al. Association of retinal thickness and optic disc-to-fovea angle to axial length of young healthy eyes. Clin Ophthalmol 2015;9:2235-41.

20 Anderson DR, Patella VM. Automated static perimetry. St. Louis, MO: Mosby, 1999: 121-90.

21 Magalhães PJ, Ram SJ, Magalhães P. Image processing with ImageJ. Biophotonics international 2004;11:36-42.

22 Weinreb RN, Khaw PT. Primary open-angle glaucoma. Lancet 2004;363:1711-20.

23 Kwon $\mathrm{YH}$, Fingert JH, Kuehn $\mathrm{MH}$, et al. Primary open-angle glaucoma. N Engl J Med 2009;360:1113-24.

24 Quigley HA. Open-Angle glaucoma. N Engl J Med 1993;328:1097-106.

25 Blumenthal EZ, Williams JM, Weinreb RN, et al. Reproducibility of nerve fiber layer thickness measurements by use of optical coherence tomography. Ophthalmology 2000;107:2278-82.

26 Schuman JS, Pedut-Kloizman T, Hertzmark E, et al. Reproducibility of nerve fiber layer thickness measurements using optical coherence tomography. Ophthalmology 1996;103:1889-98.

27 Tan O, Li G, Lu AT-H, et al. Mapping of macular substructures with optical coherence tomography for glaucoma diagnosis. Ophthalmology 2008;115:949-56.

28 Mwanza J-C, Oakley JD, Budenz DL, et al. Macular ganglion cell-inner plexiform layer: automated detection and thickness reproducibility with spectral domain-optical coherence tomography in glaucoma. Invest Ophthalmol Vis Sci 2011;52:8323-9.

29 Chauhan BC, Burgoyne CF. From clinical examination of the optic disc to clinical assessment of the optic nerve head: a paradigm change. Am J Ophthalmol 2013:156:218-27.

30 Mayama C, Saito H, Hirasawa $\mathrm{H}$, et al. Diagnosis of early-stage glaucoma by Grid-Wise macular inner retinal layer thickness measurement and effect of compensation of Disc-Fovea Inclination. Invest Ophthalmol Vis Sci 2015;56:5681-90.

31 Hirasawa $\mathrm{H}$, Araie M, Tomidokoro A, et al. Reproducibility of Circumpapillary retinal nerve fiber layer thickness measurements evaluated by circle and annulus area with and without correction for ocular rotation. J Glaucoma 2016;25:e12-18.

32 Von Noorden GK, Campos EC. Binocular vision and ocular motility. St. Louis, MO: Mosby, 2001: 127-33. 